\title{
On the existence of asymptotically almost periodic solutions for nonlinear systems
}

\author{
Juan Song ${ }^{1,2}$, Jianzhi CaO ${ }^{2 *}$ and Xiong $\mathrm{Li}^{2}$
}

\section{"Correspondence:}

cjz2004987@163.com

${ }^{2}$ School of Mathematical Sciences,

Beijing Normal University, Beijing,

Beijing 100875, P.R. China

Full list of author information is

available at the end of the article

\begin{abstract}
In this paper, a nonlinear differential equation $x^{\prime}=A(t, x)+f(t)$ is considered. Some new sufficient conditions for the existence of a bounded solution and an asymptotically almost periodic solution, which generalize and improve the previously known results, are established by using a dissipative-type condition for $A(t, x)$. Finally, an example is presented to illustrate the feasibility and effectiveness of the new results.
\end{abstract}

\section{Introduction}

In recent years, almost periodic solutions and their various generalizations have attracted the attention of many researchers (see [1-11] and the references therein). The existence of a bounded solution and an asymptotically almost periodic solution are two important properties which have a close relation to the applications of neural networks, epidemiology, etc., so they have been widely studied. For example, Medvedev [9] gave a sufficient condition to guarantee the existence of a bounded solution of the following equation:

$$
x^{\prime}=A(t, x)+f(t)
$$

where $A(t, x) \in C\left(\mathbb{R} \times \mathbb{R}^{n}, \mathbb{R}^{n}\right), f(t) \in C\left(\mathbb{R}, \mathbb{R}^{n}\right)$ and $\mathbb{R}=(-\infty,+\infty), \mathbb{R}^{n}$ denotes an Euclidean $n$-space, for $x \in \mathbb{R}^{n},\|x\|$ is any convenient norm of $x$. Using this result, he also proved the existence of periodic and almost periodic solutions when $A(t, x)$ and $f(t)$ are periodic or almost periodic in $t$ uniformly for $x$ in a bounded subset of $\mathbb{R}^{n}$. Shigeo and Masato [4] extended the existence result in [9] by using a dissipative-type condition for $A(t, x)$. Thanh and Nguyen Truong [10] considered the following difference equation:

$$
x(n+1)=\mathcal{A} x(n)+f(n), \quad n \in \mathbb{N},
$$

where $\mathbb{N}$ is a natural number and $\mathcal{A}$ is a bounded linear operator on a Banach space, the sequences $\{x(n)\}_{n \in \mathbb{N}}$ are totally ergodic, $\sigma_{\Gamma}:=\sigma(\mathcal{A}) \cap \Gamma$ is countable and the sequence $\{f(n)\}_{n \in \mathbb{N}}$ is asymptotically almost periodic, then the sequence $\{x(n)\}_{n \in \mathbb{N}}$ is asymptotically almost periodic. As an application, they studied a similar problem for an evolution equation of the form

$$
x^{\prime}=A(t) x+f(t), \quad t \in \mathbb{R}^{+},
$$

\section{Springer}

( 2013 Song et al.; licensee Springer. This is an Open Access article distributed under the terms of the Creative Commons Attribution License (http://creativecommons.org/licenses/by/2.0), which permits unrestricted use, distribution, and reproduction in any medium, provided the original work is properly cited. 
where $\mathbb{R}^{+}=[0,+\infty)$ and $A(t)$ is a linear operator on a Banach space, which is periodic, and $f(t)$ is asymptotically almost periodic. They showed a bounded mild solution $x$ is asymptotically almost periodic.

Motivated by the aforementioned discussion, in this paper, we consider the following system:

$$
x^{\prime}=A(t, x)+f(t),
$$

where $A(t, x) \in C\left(\mathbb{R}^{+} \times \mathbb{R}^{n}, \mathbb{R}^{n}\right)$ and $f(t) \in C\left(\mathbb{R}^{+}, \mathbb{R}^{n}\right)$. By employing the dissipative-type condition for $A(t, x)$, when $A(t, x)$ and $f(t)$ are asymptotically almost periodic functions, we present some new criteria ensuring the existence of a bounded solution and an asymptotically almost periodic solution of Eq. (1.4). The remaining part is organized as follows. In the next section, we introduce some definitions and lemmas. In Section 3, we obtain two theories, which guarantee the existence of a bounded solution and an asymptotically almost solution of Eq. (1.4). In Section 4, a numerical simulation is carried out to illustrate the main results.

\section{Preliminaries}

Firstly, to establish our main results, it is necessary to make the following assumptions:

$\left(C_{1}\right) f(t) \in C\left(\mathbb{R}^{+} ; \mathbb{R}^{n}\right)$ and

$$
\|f(t)+A(t, 0)\| \leq N \quad\left(\text { for all } t \in \mathbb{R}^{+}\right)
$$

where $N$ is a positive constant;

$\left(C_{2}\right) p(t) \in C\left(\mathbb{R}^{+}, \mathbb{R}\right)$. Suppose that there exist positive constants $\delta, \gamma, T_{0}$ such that

$$
p(t) \leq-\delta \quad\left(t \in\left[0, T_{0}\right]\right)
$$

and

$$
\lim _{t \rightarrow+\infty} \frac{1}{t-s} \int_{s}^{t} p(\sigma) d \sigma=-\gamma \quad \text { (uniformly for } s \geq T_{0} \text { ). }
$$

And for all $(t, x),(t, y) \in \mathbb{R}^{+} \times \mathbb{R}^{n}$,

$$
[x-y, A(t, x)-A(t, y)] \leq p(t)\|x-y\|,
$$

where $[$,$] is defined as follows (see Definition 2.5).$

We now give some definitions which can be found in [3] and [6].

Definition 2.1 If for any $\epsilon>0$, there exists a positive number $L(\epsilon)$ such that any interval of length $L(\epsilon)$ contains a $\tau$ for which

$$
\|f(t+\tau)-f(t)\| \leq \epsilon
$$

for all $t \in \mathbb{R}$, then $f(t)$ is said to be an almost periodic function. 
Definition 2.2 If for any $\epsilon>0$ and any compact set $S$ in $\mathbb{R}^{n}$, there exists a positive number $L(\epsilon, S)$ such that any interval of length $L(\epsilon, S)$ contains a $\tau$ for which

$$
\|A(t+\tau, x)-A(t, x)\| \leq \epsilon
$$

for all $t \in \mathbb{R}$ and all $x \in S$, then $A(t, x)$ is said to be almost periodic in $t$ uniformly for $x \in \mathbb{R}^{n}$.

Definition 2.3 If $f \in C\left(\mathbb{R}^{+}, \mathbb{R}\right)$ and $f(t)=g(t)+\alpha(t)$ in $\mathbb{R}^{+}, g(t)$ is an almost periodic function in $\mathbb{R}$ and $\alpha(t)$ is continuous in $\mathbb{R}^{+}, \lim _{t \rightarrow+\infty} \alpha(t)=0$, then $f(t)$ is called an asymptotically almost periodic function on $\mathbb{R}^{+}$.

Definition 2.4 If $A(t, x) \in C\left(\mathbb{R}^{+} \times \mathbb{R}^{n}, \mathbb{R}^{n}\right)$ and $A(t, x)=B(t, x)+\beta(t, x)$ in $\mathbb{R}^{+} \times \mathbb{R}^{n}$, and $B(t, x)$ is an almost periodic function in $t$ uniformly on $x \in \mathbb{R}^{n}$ and $\beta(t, x)$ is continuous in $\mathbb{R}^{+} \times \mathbb{R}^{n}, \lim _{t \rightarrow+\infty} \beta(t, x)=0$ uniformly on $x \in H \subset \Omega$, where $\Omega$ is an open set on $\mathbb{R}^{n}$ and $H$ is a compact set, then $A(t, x)$ is said to be an asymptotically almost periodic function in $t$.

Definition 2.5 Functional $[]:, \mathbb{R}^{n} \times \mathbb{R}^{n} \rightarrow \mathbb{R}$ :

$$
[x, y]=\lim _{h \rightarrow 0+} h^{-1}(\|x+h y\|-\|x\|) .
$$

The following lemma on the functional [, ] is well known (see [6]).

Lemma 2.1 [6] Let $x, y$ and $z$ be in $\mathbb{R}^{n}$. Then the functional [, ] has the following properties:

(1) $[x, y]=\inf _{h>0} h^{-1}(\|x+h y\|-\|x\|)$;

(2) $|[x, y]| \leq\|y\|$;

(3) $[x, y+z] \leq[x, y]+[x, z]$;

(4) Let $u$ be a function from a real interval $J$ into $\mathbb{R}^{n}$ such that $u^{\prime}\left(t_{0}\right)$ exists for an interior point of $J$. Then $D_{+}\left\|u\left(t_{0}\right)\right\|$ exists and

$$
D_{+}\left\|u\left(t_{0}\right)\right\|=\left[u\left(t_{0}\right), u^{\prime}\left(t_{0}\right)\right],
$$

where $D_{+}\left\|u\left(t_{0}\right)\right\|$ denotes the right derivative of $\|u(t)\|$ at $t_{0}$.

Lemma 2.2 $f(t) \in C\left(\mathbb{R}^{+}, \mathbb{R}^{n}\right)$ is an asymptotically almost periodic function if and only if for any $\epsilon>0$, there exist positive numbers $L(\epsilon)$ and $T(\epsilon)$ such that any interval of length $L(\epsilon)$ contains an $\omega$ such that when $t \geq T(\epsilon)$,

$$
\|f(t+\omega)-f(t)\|<\epsilon .
$$

Lemma 2.3 [4] Suppose that $\left(C_{2}\right)$ is satisfied. Let $u(t)$ and $v(t)$ be solutions of $(1.1)$ on an interval $[a, b)$. Then

$$
\|u(t)-v(t)\| \leq\|u(a)-v(a)\| e^{\int_{a}^{t} p(\sigma) d \sigma}
$$

for all $t \in[a, b)$. 
In order to obtain our main results, we should prove the following lemma.

Lemma 2.4 Suppose that $\left(C_{2}\right)$ is satisfied. Then

$$
\int_{T_{0}}^{t} p(\sigma) d \sigma \longrightarrow-\infty \text { as } t \longrightarrow+\infty
$$

and

$$
\sup \left\{\int_{T_{0}}^{t} e^{\int_{s}^{t} p(\sigma) d \sigma} d s ; t \geq T_{0}\right\}<+\infty
$$

Proof It follows from $\left(C_{2}\right)$ that there exists a $T_{1}>T_{0}$ such that

$$
\left|\frac{\int_{T_{0}}^{t} p(\sigma) d \sigma}{t-T_{0}}+\gamma\right|<\frac{\gamma}{2} \quad\left(t \geq T_{1}\right)
$$

then

$$
\int_{T_{0}}^{t} p(\sigma) d \sigma<-\frac{\gamma}{2}\left(t-T_{0}\right) \quad \text { for all } t \geq T_{1} .
$$

This means that

$$
\int_{T_{0}}^{t} p(\sigma) d \sigma \leq-\frac{\gamma}{2}\left(t-T_{0}\right) \longrightarrow-\infty \quad \text { as } t \rightarrow+\infty
$$

Since

$$
\int_{s}^{t} p(\sigma) d \sigma \leq-\frac{\gamma}{2}(t-s) \quad \text { for } t>s \geq T_{1}
$$

for each $t>T_{1}$, we have

$$
\begin{aligned}
\int_{T_{0}}^{t} e^{\int_{s}^{t} p(\sigma) d \sigma} d s & =\int_{T_{0}}^{T_{1}} e^{\int_{s}^{T_{1}} p(\sigma) d \sigma} e^{\int_{T_{1}}^{t} p(\sigma) d \sigma} d s+\int_{T_{1}}^{t} e^{t} p(\sigma) d \sigma d s \\
& \leq \int_{T_{0}}^{T_{1}} e^{\int_{s}^{T_{1}} p(\sigma) d \sigma} d s+\int_{T_{1}}^{t} e^{-\frac{\gamma}{2}(t-s)} d s \\
& =\int_{T_{0}}^{T_{1}} e^{\int_{s}^{T_{1}} p(\sigma) d \sigma} d s+\frac{2}{\gamma}\left[1-e^{-\frac{\gamma}{2}\left(t-T_{1}\right)}\right] .
\end{aligned}
$$

Therefore,

$$
\begin{aligned}
\int_{T_{0}}^{t} e^{\int_{s}^{t} p(\sigma) d \sigma} d s & \leq \frac{2}{\gamma}+\int_{T_{0}}^{T_{1}} e^{p_{0}\left(T_{1}-s\right)} d s \\
& =\frac{1}{p_{0}}\left(e^{p_{0}\left(T_{1}-T_{0}\right)}-e^{0}\right)+\frac{2}{\gamma} \\
& \leq \frac{1}{p_{0}} e^{p_{0}\left(T_{1}-T_{0}\right)}+\frac{2}{\gamma}
\end{aligned}
$$


for all $t \geq T_{1}$ and $p_{0}=\sup \left\{|p(t)| ; t \in\left[T_{0}, T_{1}\right]\right\}$. Then

$$
\sup \left\{\int_{T_{0}}^{t} e^{\int_{s}^{t} p(\sigma) d \sigma} ; t \geq T_{0}\right\}<+\infty
$$

This completes the proof.

\section{Existence of bounded solutions and asymptotically periodic solutions}

In this section, it will be shown that, under certain conditions, the system (1.4) has a bounded solution and an asymptotically periodic solution.

Theorem 3.1 Suppose that conditions $\left(C_{1}\right),\left(C_{2}\right)$ are satisfied. Let $r$ be defined as

$$
r=\Gamma\left(r_{0}+N\right),
$$

where

$$
\Gamma=\max \left\{1, \sup _{T_{0} \leq s \leq t} e^{\int_{s}^{t} p(\sigma) d \sigma}, \sup _{T_{0} \leq t} \int_{T_{0}}^{t} e^{\int_{s}^{t} p(\sigma) d \sigma} d s\right\}
$$

and

$$
r_{0}=\frac{N}{\delta} .
$$

Then Eq. (1.4) has a bounded solution $u(t)$ on $\mathbb{R}^{+}$such that $\|u(t)\| \leq r$ for $t \in \mathbb{R}^{+}$. Furthermore, if $v(t)$ is any solution of Eq. (1.4), then $\|u(t)-v(t)\| \rightarrow 0$ as $t \rightarrow+\infty$.

Proof If $A(t, 0) \not \equiv 0$ for $t \in \mathbb{R}^{+}$, we replace $A(t, x)$ and $f(t)$ by $A(t, x)-A(t, 0)$ and $f(t)+$ $A(t, 0)$, respectively. We assume, henceforth, that $A(t, 0) \equiv 0$ and $\|f(t)\| \leq N$ for all $t \in \mathbb{R}^{+}$ and fix a vector $u_{0} \in \mathbb{R}^{n}$ with $\left\|u_{0}\right\|=r_{0}$. For each positive integer $n$ with $n>T_{0}>\frac{1}{n}$, we consider the following Cauchy problem:

$$
x^{\prime}=A(t, x)+f(t), \quad x\left(\frac{1}{n}\right)=u_{0} .
$$

We find that the conditions $\left(V_{1}\right)-\left(V_{3}\right)$ and $\left(K_{1}\right)-\left(K_{4}\right)$ in [5] can be satisfied by $\left(C_{1}\right),\left(C_{2}\right)$ in the present paper, then Corollary 5.1 in [5] can now be applied to guarantee the (c.p) has a unique solution $u_{n}$ on $\left[\frac{1}{n}, n\right]$. We first prove that

$$
\left\|u_{n}(t)\right\| \leq r_{0} \quad \text { for all } t \in\left[\frac{1}{n}, T_{0}\right]
$$

In fact, otherwise there exists some $t_{1}$ such that $\left\|u_{n}\left(t_{1}\right)\right\|=r_{1}$, where $r_{1}$ is an arbitrary number such that $r_{1}>r_{0}$. Let $\tau=\sup \left\{t \in\left[\frac{1}{n}, t_{1}\right] ;\left\|u_{n}(t)\right\| \leq r_{1}\right\}$, by the continuity of $u_{n}(t)$, it follows easily that $\frac{1}{n}<\tau \leq T_{0}$. Then $\tau<T_{0}$ implies $\left\|u_{n}(\tau)\right\|=r_{1}$, and by Lemma 2.1 and 
$\left(C_{2}\right)$, we have

$$
\begin{aligned}
D_{+}\left\|u_{n}(\tau)\right\| & =\left[u_{n}(\tau), u_{n}^{\prime}(\tau)\right] \\
& =\left[u_{n}(\tau), A\left(\tau, u_{n}(\tau)\right)+f(\tau)\right] \\
& \leq\left[u_{n}(\tau), A\left(\tau, u_{n}(\tau)\right)\right]+\left[u_{n}(\tau), f(\tau)\right] \\
& \leq p(\tau)\left\|u_{n}(\tau)\right\|+\|f(\tau)\| \\
& \leq p(\tau) r_{1}+N .
\end{aligned}
$$

For each $\epsilon>0$, there exists an $h_{0}>0$ such that

$$
\begin{aligned}
\left\|u_{n}(\tau+h)\right\| & \leq\left\|u_{n}(\tau)\right\|+h\left(p(\tau) r_{1}+N+\epsilon\right) \\
& =r_{1}+h\left(p(\tau) r_{1}+N+\epsilon\right)
\end{aligned}
$$

for $0<h \leq h_{0}$. Since $r_{1}>r_{0}=\frac{N}{\delta}, p(\tau) r_{1} \leq-\delta r_{1}<-N, p(\tau) r_{1}+N<0$. Thus, for $\epsilon$ with $0<\epsilon<-\left(p(\tau) r_{1}+N\right)$, there exists a sufficiently small $h>0$ such that $\left\|u_{n}(\tau+h)\right\|<r_{1}$. This contradicts the definition of $\tau$. Then $\left\|u_{n}(t)\right\| \leq r_{0}$ for all $t \in\left[\frac{1}{n}, T_{0}\right]$.

On the other hand, using the following differential inequality:

$$
D_{+}\left\|u_{n}(t)\right\| \leq p(t)\left\|u_{n}(t)\right\|+\|f(t)\| \quad \text { for } t \in\left[T_{0}, n\right],
$$

we have

$$
\begin{aligned}
\left\|u_{n}(t)\right\| & \leq\left\|u_{n}\left(T_{0}\right)\right\| e^{\int_{T_{0}}^{t} p(\sigma) d \sigma}+\int_{T_{0}}^{t} N e^{\int_{s}^{t} p(\sigma) d \sigma} d s \\
& \leq \Gamma\left(r_{0}+N\right)=r .
\end{aligned}
$$

It thus follows that $\left\|u_{n}(t)\right\| \leq r$ for all $t \in\left[\frac{1}{n}, n\right]$. Lemma 8.1 in [8] can now be applied to guarantee the existence on $(0,+\infty)$ of a bounded solution $u(t)$ of Eq. (1.4). By the continuity of $u(t), u(t)$ is a bounded solution on $\mathbb{R}^{+}$which also satisfies $\|u(t)\| \leq r$. If $v(t)$ is any solution of Eq. (1.4) on $\mathbb{R}^{+}$, by Lemma 2.3, we have

$$
\begin{aligned}
\|u(t)-v(t)\| & \leq\|u(0)-v(0)\| e^{\int_{0}^{t} p(\sigma) d \sigma} \\
& =\|u(0)-v(0)\| e^{\int_{0}^{T_{0}} p(\sigma) d \sigma} \cdot e^{\int_{T_{0}}^{t} p(\sigma) d \sigma} .
\end{aligned}
$$

By Lemma 2.4, we obtain $e^{\int_{T_{0}}^{t} p(\sigma) d \sigma} \rightarrow 0$, when $t \rightarrow+\infty, \int_{T_{0}}^{t} p(\sigma) d \sigma \rightarrow-\infty$, and then $\|u(t)-v(t)\| \rightarrow 0$ as $t \rightarrow+\infty$. This completes the proof.

Theorem 3.2 Suppose that $A(t, x)$ is asymptotically almost periodic in $t$ uniformly for $x \in$ $\operatorname{Sr}(0)$, where $r$ is a positive number defined by Eq. (3.1) and $\operatorname{Sr}(0)=\left\{x \in \mathbb{R}^{n} ;\|x\| \leq r\right\}$, and $f(t)$ is an asymptotically almost periodic function. Suppose, furthermore, that the condition $\left(C_{2}\right)$ is satisfied. Then Eq. (1.4) has an asymptotically almost periodic solution on $\mathbb{R}^{+}$.

Proof First, we prove that $f(t)$ is bounded. $f(t)=g(t)+\alpha(t)$ in $\mathbb{R}^{+}$, and $g(t)$ is an almost periodic function in $\mathbb{R}$. For any $\varepsilon \leq 1$, there is an $l(\varepsilon)>0$, when $t \in[0, l(\varepsilon)]$, there is an $M>$ 0 , $\|g(t)\| \leq M$. For any $t \in \mathbb{R}$, choose $\tau \in[-t,-t+l(\varepsilon)]$, then $t+\tau \in[0, l(\varepsilon)],\|g(t+\tau)\|<M$ and $\|g(t+\tau)-g(t)\|<1$, so for any $t,\|g(t)\|<M+1$. While $\alpha(t) \rightarrow 0(t \rightarrow \infty)$, we have a 
positive $N>0$ such that $\|f(t)\|<N$, then the condition $\left(C_{1}\right)$ is satisfied. Conditions $\left(C_{1}\right)$ and $\left(C_{2}\right)$ are satisfied, let $u(t)$ be a bounded solution of (1.4) on $\mathbb{R}^{+}$obtained in Theorem 3.1. Note that $\|u(t)\| \leq r$ for all $t \in \mathbb{R}^{+}$, where $r$ is a number defined by Eq. (3.1).

Notice that $B(t, x)+g(t)$ is also an almost periodic function in $t$ uniformly for $x \in \operatorname{Sr}(0)$. For each $\epsilon>0$, there exist a positive number $t_{1}(\epsilon, \operatorname{Sr}(0))$ and a positive number $L(\epsilon, \operatorname{Sr}(0))$ such that any interval of length $L(\epsilon, \operatorname{Sr}(0))$ contains an $\omega$,

$$
\begin{aligned}
& \|A(t+\omega, x)-A(t, x)+f(t+\omega)-f(t)\| \\
& \leq\|B(t+\omega, x)+g(t+\omega)-B(t, x)-g(t)\| \\
& \quad+\|\beta(t+\omega, x)-\beta(t, x)\|+\|\alpha(t+\omega)-\alpha(t)\| \\
& \leq \epsilon \text { for all } t>t_{1}(\epsilon, \operatorname{Sr}(0)) \text { and } x \in \operatorname{Sr}(0) .
\end{aligned}
$$

By Lemma 2.1, $\left(C_{2}\right)$ and Eq. (3.4), we have

$$
\begin{aligned}
D_{+} & \|u(t+\omega)-u(t)\| \\
= & {[u(t+\omega)-u(t), A(t+\omega, u(t+\omega))+f(t+\omega)-(A(t, u(t))+f(t))] } \\
\leq & {[u(t+\omega)-u(t), A(t, u(t+\omega))-A(t, u(t))] } \\
& \quad+\|A(t+\omega, u(t+\omega))-A(t, u(t+\omega))+f(t+\omega)-f(t)\| \\
\leq & p(t)\|u(t+\omega)-u(t)\|+\epsilon
\end{aligned}
$$

for all $t>t_{1}$. Solving this differential inequality, we have

$$
\begin{aligned}
\|u(t+\omega)-u(t)\| & \leq\|u(t-\eta+\omega)-u(t-\eta)\| e^{\int_{t-\eta}^{t} p(\sigma) d \sigma}+\epsilon \int_{t-\eta}^{t} e^{\int_{s}^{t} p(\sigma) d \sigma} d s \\
& \leq 2 r e^{\int_{t-\eta}^{t} p(\sigma) d \sigma}+\epsilon \int_{t-\eta}^{t} e^{\int_{s}^{t} p(\sigma) d \sigma} d s
\end{aligned}
$$

where $\eta$ is a positive number to be chosen later appropriately, and $t-\eta \geq 0$. We show that

$$
K=\sup \left\{\int_{t-\eta}^{t} e^{\int_{s}^{t} p(\sigma) d \sigma} d s ; t \geq t_{1}, t-\eta \geq 0\right\}
$$

is finite. In fact, this follows from the following estimates:

$$
\int_{t-\eta}^{t} e^{\int_{s}^{t} p(\sigma) d \sigma} d s \leq \begin{cases}\left(1+\frac{1}{\delta}\right) \Gamma, & t \geq T_{0}, t-\eta \leq T_{0} \\ \Gamma, & t \geq T_{0}, t-\eta \geq T_{0}\end{cases}
$$

Let $T_{1}>T_{0}$ be a number such that

$$
\int_{s}^{t} p(\sigma) d \sigma \leq-\frac{\gamma}{2}(t-s) \quad\left(t \geq T_{1}, s \geq T_{0}\right)
$$

and

$$
e^{-\gamma T_{1}}<\frac{\epsilon}{2 \Gamma}
$$

We will show that $\|u(t+\omega)-u(t)\| \leq K_{0} \epsilon$, where $K_{0}$ is a positive constant independent of $\epsilon$ and $\omega$. 
We must estimate $e^{\int_{t-\eta}^{t} p(\sigma) d \sigma}$ for $t$ large enough.

Since $e^{\int_{t-2 T_{1}}^{t} p(\sigma) d \sigma} \leq e^{-\frac{\gamma}{2}\left(t-t+2 T_{1}\right)}=e^{-\gamma T_{1}}\left(\right.$ when $\left.t-2 T_{1} \geq T_{0}\right)$, if $t-\eta \geq T_{0}, t-4 T_{1} \geq t-\eta$, $t \geq T_{1}, t-4 T_{1} \geq T_{0}$, then

$$
\begin{aligned}
e^{\int_{t-\eta}^{t} p(\sigma) d \sigma} & =e^{\int_{t-\eta}^{t-2 T_{1}} p(\sigma) d \sigma} e^{\int_{t-2 T_{1}}^{t} p(\sigma) d \sigma} \\
& \leq \Gamma e^{-\gamma T_{1}} .
\end{aligned}
$$

When we choose $\eta=4 T_{1}$ and $t-4 T_{1} \geq T_{0}$, that is, $t \geq 4 T_{1}+T_{0}$, then, for any $\epsilon>0$, there exists a positive number $L(\epsilon, \operatorname{Sr}(0))$ such that any interval of length $L(\epsilon, \operatorname{Sr}(0))$ contains an $\omega$, when $t \geq T=\max \left\{t_{1}(\epsilon, \operatorname{Sr}(0)), 4 T_{1}+T_{0}\right\}, \eta=4 T_{1}, K_{0}=\gamma+K$,

$$
\begin{aligned}
\|u(t+\omega)-u(t)\| & \leq 2 r \cdot \Gamma e^{-\gamma T_{1}}+\epsilon K \\
& \leq r \epsilon+K \epsilon=(r+K) \epsilon=K_{0} \epsilon .
\end{aligned}
$$

From Lemma 2.2, $u(t)$ is an asymptotically almost periodic solution of Eq. (1.4). This completes the proof.

Remark 3.1 In [4], employing the dissipative-type condition for $A(t, x)$, the authors gave some sufficient conditions to prove the existence of a bounded solution, a periodic or almost periodic solution of the equation $x^{\prime}=A(t, x)+f(t)$. Extension of this result has been obtained in one direction: from periodic and almost periodic to asymptotically almost periodic forcing. The equation can be more widely used with asymptotically almost periodic functions.

Remark 3.2 The condition $\left(C_{2}\right)$ implies the following hypothesis.

$\left(C_{2}^{\prime}\right)$ Suppose that there exist $p(t) \in\left(\mathbb{R}^{+}, \mathbb{R}\right)$ and positive constants $\delta, \delta_{1}, T_{0}$ and $T_{1}$ such that

$$
\begin{aligned}
& p(t) \leq-\delta \quad\left(t \in\left[0, T_{0}\right]\right), \\
& p(t) \leq-\delta_{1} \quad\left(t \in\left[T_{1},+\infty\right]\right) .
\end{aligned}
$$

And for all $(t, x),(t, y) \in \mathbb{R}^{+} \times \mathbb{R}^{n}$,

$$
[x-y, A(t, x)-A(t, y)] \leq p(t)\|x-y\| .
$$

We know that $\left(C_{2}^{\prime}\right)$ can also be used to prove the lemmas in Section 2 and the theorems in Section 3 leaving the conclusion unchanged. $\left(C_{2}^{\prime}\right)$ as well as $\left(C_{2}\right)$ yields the existence of a bounded solution, and the process of the proof is similar to the proof before, and we need not necessarily do it again.

\section{The example}

In this section, we give a numerical example to illustrate the conditions required in our theorems. We construct the following differential equation:

$$
x^{\prime}=-x(\sin t+\sin \sqrt{2} t+3)+\sin \frac{1}{(1+t)^{2}}-(\sin t+\sin \sqrt{2} t+3)+\sin \frac{1}{(1+t)^{2}},
$$




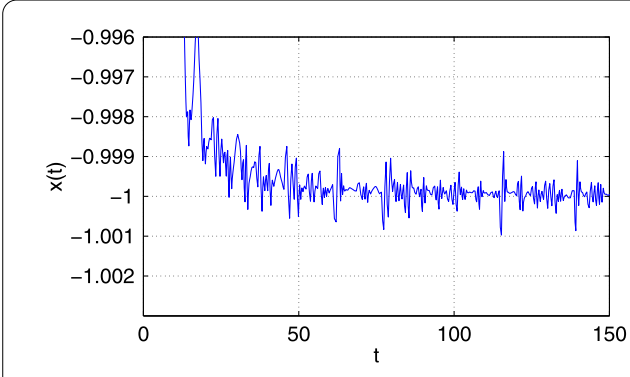

(a)

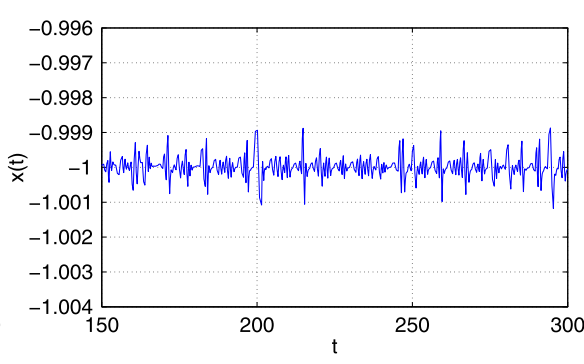

(b)

Figure 1 Trajectory graphs of the system (4.1) with initial value $x(0)=-0.1$. (a), (b) are the trajectory graphs of the simulation time $0-150$ and 150-300, respectively.

where $A(t, x)=-x(\sin t+\sin \sqrt{2} t+3)+\sin \frac{1}{(1+t)^{2}}$ is an asymptotically almost periodic function in $t \in \mathbb{R}^{+}$uniformly on $x$ which belongs to a compact set and $f(t)=-(\sin t+\sin \sqrt{2} t+$ $3)+\sin \frac{1}{(1+t)^{2}}$ is an asymptotically almost periodic function on $\mathbb{R}^{+}$.

First,

$$
\|f(t)+A(t, 0)\|=\left\|-(\sin t+\sin \sqrt{2} t+3)+2 \sin \frac{1}{(t+1)^{2}}\right\| \leq 7,
$$

we know that $\left(C_{1}\right)$ is satisfied.

On the other hand, there exists $p(t)=-1$ such that

$$
\begin{aligned}
{[x} & -y, A(t, x)-A(t, y)] \\
& =\lim _{h \rightarrow 0+} \frac{\|(x-y)+h(A(t, x)-A(t, y))\|-\|x-y\|}{h} \\
& =\lim _{h \rightarrow 0+} \frac{\|(x-y)-h[(x-y)(\sin t+\sin \sqrt{2} t+3)]\|-\|x-y\|}{h} \\
& =\|x-y\| \lim _{h \rightarrow 0+} \frac{1-h(\sin t+\sin \sqrt{2} t+3)-1}{h} \\
& =-(\sin t+\sin \sqrt{2} t+3)\|x-y\| \leq-\|x-y\|=p(t)\|x-y\|
\end{aligned}
$$

and

$$
\left.\lim _{t \rightarrow+\infty} \frac{1}{t-s} \int_{s}^{t} p(\sigma) d \sigma=\lim _{t \rightarrow+\infty}(-1) \cdot \frac{t-s}{t-s}=-1 \quad \text { (uniformly for } s \geq T_{0}\right),
$$

$\left(C_{2}\right)$ is satisfied too.

Then, from Theorem 3.1 and Theorem 3.2, we get a bounded solution and an asymptotically almost periodic solution on $\mathbb{R}^{+}$of Eq. (4.1) as follows:

$$
x(t)=e^{\cos t+\frac{1}{\sqrt{2}} \cos \sqrt{2} t-3 t}\left(2 \int_{0}^{t} \sin \frac{1}{(s+1)^{2}} e^{3 s-\cos s-\frac{1}{\sqrt{2}} \cos \sqrt{2} s} d s+c\right)-1 .
$$

We show the semiflow in Figure 1. 
Authors' contributions

All authors completed the paper together. All authors read and approved the final manuscript.

\section{Author details}

'Department of Statistics and Applied Mathematics, Hubei University of Economics, Wuhan, Hubei 430205, P.R. China.

${ }^{2}$ School of Mathematical Sciences, Beijing Normal University, Beijing, Beijing 100875, P.R. China.

\section{Acknowledgements}

The authors would like to thank the two referees for their valuable suggestions and comments concerning improvement of the work.

Received: 13 October 2012 Accepted: 22 January 2013 Published: 7 February 2013

\section{References}

1. Dads, EA, Ezzinbi, K, Arino, O: Periodic and almost periodic solutions for some differential equations in Banach spaces. Nonlinear Anal., Theory Methods Appl. 31, 163-170 (1998)

2. Gao, H, Wang, K, Wei, F, Ding, X: Massera-type theorem and asymptotically periodic logistic equations. Nonlinear Anal., Real World Appl. 7, 1268-1283 (2006)

3. He, C: Almost Periodic Differential Equations. Higher Education Press, Beijing (1992) (in Chinese)

4. Shigeo, K, Masato, I: On the existence of periodic solutions and almost periodic solutions for nonlinear systems. Nonlinear Anal., Theory Methods Appl. 24, 1183-1192 (1995)

5. Shigeo, K: Some remarks on nonlinear ordinary differential equations in a Banach space. Nonlinear Anal. 5, 81-93 (1981)

6. Shigeo, K: Some remarks on nonlinear differential equations in Banach space. Hokkaido Math. J. 4, $205-226$ (1975)

7. Liu, B, Huang, L: Existence and stability of almost periodic solutions for shunting inhibitory cellular neural networks with time-varying delays. Chaos Solitons Fractals 31, 211-217 (2007)

8. Krasnosel'skii, MA: The Operator of Translation Along the Trajectories of Differential Equations. Translat. Math. Monoger., vol. 19 (1968)

9. Medvedev, NV: Certain tests for the existence of bounded solutions of systems of differential equations. Differ. Uravn (Minsk) 4, 1258-1264 (1968)

10. Thanh, N: Asymptotically almost periodic solutions on the half-line. J. Differ. Equ. Appl. 11, 1231-1243 (2005)

11. Xia, Y, Cao, J, Lin, M: New results on the existence and uniqueness of almost periodic solution for BAM neural networks with continuously distributed delays. Chaos Solitons Fractals 31, 928-936 (2007)

doi:10.1186/1687-1847-2013-28

Cite this article as: Song et al.: On the existence of asymptotically almost periodic solutions for nonlinear systems.

Advances in Difference Equations 2013 2013:28.

\section{Submit your manuscript to a SpringerOpen ${ }^{\ominus}$ journal and benefit from:}

- Convenient online submission

- Rigorous peer review

- Immediate publication on acceptance

Open access: articles freely available online

High visibility within the field

- Retaining the copyright to your article 